\title{
BCS-BEC crossover in dense relativistic matter: Collective excitations
}

\author{
Tomáš Brauner* \\ Institut für Theoretische Physik, J. W. Goethe-Universität, \\ Max von Laue-Str. 1, 60438 Frankfurt am Main, Germany ${ }^{\dagger}$
}

\begin{abstract}
We study the relativistic BCS-BEC crossover within a class of Nambu-Jona-Lasinio type models, including arbitrary Lorentz-scalar pairing channels. Using the mean-field approximation we investigate spectral properties of the collective bosonic excitations in the superfluid phase, with particular attention to the Nambu-Goldstone bosons of the broken symmetry. This is a first step towards a systematic improvement of the mean-field approximation by including the fluctuation effects. The general results are illustrated on pairing in dense two-flavor quark matter — the two-flavor color superconductor.
\end{abstract}

PACS numbers: 11.30.Qc, 11.10.Wx, 25.75.Nq

Keywords: BCS-BEC crossover, Nambu-Jona-Lasinio model, Spontaneous symmetry breaking, Type-II Goldstone bosons.

\section{INTRODUCTION}

It was noted long ago that Bardeen-Cooper-Schrieffer (BCS) superconductivity of correlated Cooper pairs and Bose-Einstein condensation (BEC) of tightly bound difermion molecules are merely two sides of the same coin. Eagles [1] and Leggett [2] were perhaps the first to observe that a many-fermion system can display both the BCS and the BEC behavior, and these two regimes are connected by a smooth crossover as the strength of the attractive interaction between the fermions is varied. The crossover can be described at least qualitatively well by the conventional mean-field approximation provided one determines the chemical potential self-consistenly by fixing the overall particle density.

However, it was only recently that the BCS-BEC crossover could be realized experimentally using ultracold atomic Fermi gases [3, 4, 5, 6], where the strength of the interaction can be tuned by external magnetic field and thereby, using the Feshbach resonance, cover the whole range between the BCS and BEC limits. The dilute atomic gases represent particularly clean and well controlled examples of interacting many-body systems. It is believed that they may provide an ideal theoretical playground for development of many-body techniques, that would be later applied to more complicated strongly correlated systems.

The mean-field theory of Eagles and Leggett was for the first time improved by Nozières and Schmitt-Rink 7] who included the contribution of the pair fluctuations to the total particle density. In the past decade, various extensions of the mean-field approximation have been proposed, including boson-fermion models 8 as well as purely fermionic self-consistent schemes [9, 10]. An extensive list of references may be found in the re-

\footnotetext{
$\dagger$ On leave from Department of Theoretical Physics, Nuclear Physics Institute ASCR, 25068 Rež, Czech Republic

*Electronic address: brauner@ujf.cas.cz
}

views [11, 12]. The most recently developed techniques are compared in [13]. Simultaneously to the analytic approximation schemes, the interacting Fermi gas is also being studied using numerical Monte Carlo simulations [14, 15].

In high-energy physics, the analogy with BCS superconductivity was used long ago by Nambu and JonaLasinio [16, 17] to propose a model for dynamical chiral symmetry breaking. Later, Cooper pairing of two fermions near their Fermi surface found an application in the physics of dense nuclear/quark matter, giving rise in particular to the phenomenon of color superconductivity [18, 19]; see [20] for the most recent review. The crossover physics is relevant for these high-energy systems because of the strong-coupling nature of quantum chromodynamics. Concretely, the so-called pseudogap phase as a precursor to superfluidity of tightly bound Cooper pairs was investigated in models of chiral symmetry breaking [21, 22, 23, 24] as well as color superconductivity [25]. The structural change of Cooper pairs with increasing coupling strength and the possibility of their BEC were studied in [26, 27, 28, 29]. The spectrum of diquarks in various color-superconducting phases was investigated by Ebert et al. [30, 31].

The actual crossover between the BCS and BEC regimes in dense quark matter in dependence on the coupling strength has started to be studied only recently 32, 33, 34]. Perhaps the first attempts to go beyond the mean-field approximation have been made in Refs. [35, 36, 37]. In particular, Abuki [36] has conducted an extensive study of fluctuation effects in the normal phase, using the Gaussian approximation. The goal of this paper is to investigate the spectrum of the bosonic collective modes below the critical temperature. Thereby we construct a framework for description of fluctuation effects in the superfluid phase.

The plan of the paper is following. In the next section, we derive the general formulas valid for a class of models of the Nambu-Jona-Lasinio (NJL) type, that describe (even-parity) spin-zero pairing in relativistic fermion matter. The model of Abuki [36], which is a 
special case, is then investigated in detail in the following section. In particular, we study the spectral properties of the bosonic collective modes in the mean-field approximation. Possible extensions of the present work are discussed in the conclusions.

\section{NJL MODELS OF PAIRING}

\section{A. Model definition}

We consider the general class of models defined by the Lagrangian

$$
\mathcal{L}=\bar{\psi}\left(i \not \partial+\mu \gamma_{0}-m\right) \psi+\frac{G}{4} \sum_{a}\left(\psi^{T} T_{a} \psi\right)\left(\bar{\psi} \bar{T}_{a} \bar{\psi}^{T}\right),
$$

where $\bar{T}_{a}=\gamma_{0} T_{a}^{\dagger} \gamma_{0}$. The spinor $\psi$ denotes a set of fermion flavors with common mass $m$ and chemical potential $\mu$, while $T_{a}$ are matrices that furnish an antisymmetric tensor representation under the symmetry transformations acting on $\psi$. In general, they act on both Dirac and flavor indices of $\psi$. Even though the Lagrangian (11) covers, in a simplified manner, a number of systems investigated in literature [38], at this stage we completely neglect correlations in the fermionantifermion channel that may lead to dynamical breaking of the chiral symmetry.

This model is analyzed using the standard HubbardStratonovich transformation. We start with the definition of the Nambu spinor $\Psi=\left(\psi, \psi^{\mathcal{C}}\right)^{T}$ [49]. Using the charge conjugate field, $\psi^{\mathcal{C}}=C \bar{\psi}^{T}$, the interaction term may be rewritten as $\frac{G}{4}\left|\bar{\psi}^{\mathcal{C}} P_{a} \psi\right|^{2}$, where $P_{a}=C^{-1} T_{a}$. It is removed from the Lagrangian by introducing new auxiliary scalar fields $\Delta_{a}$ and adding

$$
\Delta \mathcal{L}=-\frac{1}{G}\left|\Delta_{a}-\frac{G}{2} \bar{\Psi}\left(\begin{array}{cc}
0 & 0 \\
P_{a} & 0
\end{array}\right) \Psi\right|^{2} .
$$

The new, semibosonized Lagrangian reads

$$
\mathcal{L}_{\text {semi }}=-\frac{1}{G} \Delta_{a}^{*} \Delta_{a}+\frac{1}{2} \bar{\Psi} \mathcal{D}^{-1} \Psi,
$$

where

$$
\mathcal{D}^{-1}=\left(\begin{array}{cc}
i \not \partial+\gamma_{0} \mu-m & \Delta_{a} \bar{P}_{a} \\
\Delta_{a}^{*} P_{a} & i \not \partial-\gamma_{0} \mu-m
\end{array}\right),
$$

is the fermion propagator in the coordinate space. Upon integrating out the fermions, the thermodynamic potential $\Omega$ is given by

$$
\begin{aligned}
e^{-\beta \Omega} & =\int d \Delta_{a} d \Delta_{a}^{*} \exp \left(-S_{\mathrm{eff}}\left[\Delta, \Delta^{*}\right]\right), \\
S_{\mathrm{eff}} & =\frac{1}{G} \int_{0}^{\beta} d \tau \int d^{3} \mathbf{x}\left|\Delta_{a}(\mathbf{x}, \tau)\right|^{2}-\frac{1}{2} \log \operatorname{det} \mathcal{D}^{-1} .
\end{aligned}
$$

This functional integral, of course, cannot be evaluated exactly. Note that the bosonized action $S_{\text {eff includes the }}$ effect of fermion loops to all orders, due to the presence of the determinant of the inverse fermion propagator $\mathcal{D}^{-1}$. In other words, it contains all Feynman graphs with the scalar fields as external lines. It is thus to be interpreted as a classical action for the scalars $\Delta_{a}, \Delta_{a}^{*}$, and the above functional integral as the generating functional of their Green's functions (at zero source). This can be determined from the quantum effective action associated to the classical action $S_{\text {eff }}$, being the sum of all one-bosonirreducible graphs.

Since we assume that the leading nonperturbative behavior, which gives rise to the pairing, is borne by the fermion loops that have already been resummed to all orders, the expansion in the number of bosonic loops provides a natural approximation scheme for the calculation of the thermodynamic potential. The tree level corresponds to simply setting $\beta \Omega_{\mathrm{MF}}=S_{\text {eff }}$ : This is the standard mean-field approximation. At one loop, the thermodynamic potential would be given by

$$
\beta \Omega_{1 \mathrm{~L}}=\beta \Omega_{\mathrm{MF}}+\frac{1}{2} \log \operatorname{det} \Xi,
$$

where

$$
\Xi_{a b}(X-Y)=\frac{\delta^{2} S_{\mathrm{eff}}}{\delta \Phi_{a}^{\dagger}(X) \delta \Phi_{b}(Y)} \equiv\left(\begin{array}{cc}
\chi_{a b}^{\Delta \Delta^{*}} & \chi_{a b}^{\Delta \Delta} \\
\chi_{a b}^{\Delta *} \Delta^{*} & \chi_{a b}^{\Delta *} \Delta
\end{array}\right),
$$

is the inverse propagator of the bosonic modes; $\Phi$ is the scalar doublet field, $\Phi_{a}=\left(\Delta_{a}, \Delta_{a}^{*}\right)^{T}$. Note that in the normal phase, Eq. (44) reduces to the Gaussian approximation adopted by Abuki [36]. Carrying out the indicated differentiation and setting the fields $\Delta_{a}$ equal to their vacuum expectation values (which we hereafter refer to by the same symbol), we arrive at the expressions for the normal and anomalous parts of the inverse propagators,

$$
\begin{aligned}
& \chi_{a b}^{\Delta \Delta^{*}}\left(i \Omega_{N}, \mathbf{p}\right)=\frac{1}{G} \delta_{a b}+\frac{1}{2} T \sum_{n} \int \frac{d^{3} \mathbf{k}}{(2 \pi)^{3}} \\
& \times \operatorname{Tr}\left[P_{a} \mathcal{D}^{\psi \bar{\psi}}\left(i \omega_{n}, \mathbf{k}\right) \bar{P}_{b} \mathcal{D}^{\bar{\psi} \psi}\left(i \omega_{n}-i \Omega_{N}, \mathbf{k}-\mathbf{p}\right)\right] \\
& \chi_{a b}^{\Delta \Delta}\left(i \Omega_{N}, \mathbf{p}\right)=\frac{1}{2} T \sum_{n} \int \frac{d^{3} \mathbf{k}}{(2 \pi)^{3}} \\
& \times \operatorname{Tr}\left[P_{a} \mathcal{D}^{\psi \psi}\left(i \omega_{n}, \mathbf{k}\right) P_{b} \mathcal{D}^{\psi \psi}\left(i \omega_{n}-i \Omega_{N}, \mathbf{k}-\mathbf{p}\right)\right]
\end{aligned}
$$

where $\psi$ and $\bar{\psi}$ in the superscripts to $\mathcal{D}$ denote the respective matrix elements of the matrix propagator (2). The remaining two entries of the matrix (5) follow from the general relations,

$$
\begin{aligned}
\chi_{a b}^{\Delta^{*} \Delta}(\omega, \mathbf{p}) & =\chi_{b a}^{\Delta \Delta^{*}}(-\omega,-\mathbf{p}), \\
\chi_{a b}^{\Delta \Delta}(\omega, \mathbf{p}) & =\chi_{b a}^{\Delta \Delta}(-\omega,-\mathbf{p}), \\
\chi_{a b}^{\Delta^{*} \Delta^{*}}(\omega, \mathbf{p}) & =\left[\chi_{b a}^{\Delta \Delta}\left(\omega^{*}, \mathbf{p}\right)\right]^{*} .
\end{aligned}
$$

valid for an arbitrary complex frequency $\omega$. 


\section{B. Fermion propagator}

In order to be actually able to invert the matrix $\mathcal{D}^{-1}$ (2), we now make a specific assumption about the fermion pairing pattern. We assume that the Cooper pairs are Lorentz scalars, i.e., the matrices $T_{a}$ have the form $T_{a}=$ $C \gamma_{5} Q_{a}$, where $Q_{a}$ act just on the internal symmetry indices. The order parameters $\Delta_{a}$ now enter the fermion propagator in terms of the expression $M=\Delta_{a} Q_{a}^{\dagger}$. Note that Pauli principle requires $T_{a}$ to be antisymmetric so that both $Q_{a}$ and $M$ have to be symmetric matrices.

With the above assumption, the denominators of the matrix fermion propagator depend only on the combinations $M M^{\dagger}$ and $M^{\dagger} M$. Being Hermitian and positive definite, the matrix $M M^{\dagger}$ may be spectrally decomposed as 39.

$$
M M^{\dagger}=\sum_{r} \Delta_{r}^{2} \mathcal{P}_{r}
$$

where $\mathcal{P}_{r}$ is a set of projectors on the respective eigenvectors, and $\Delta_{r}^{2}$ are the real positive eigenvalues whose relation to $\Delta_{a}$ will be clarified later. Using the symmetry of $M$, we find the equivalent decomposition,

$$
M^{\dagger} M=\sum_{r} \Delta_{r}^{2} \mathcal{P}_{r}^{*} .
$$

The parameters $\Delta_{r}$ play the role of gaps in the (fermionic) quasiparticle dispersion relations, which are found as poles in the fermion propagator $\mathcal{D}$,

$$
\begin{aligned}
E_{\mathbf{k} r}^{e} & =\sqrt{\left(\xi_{\mathbf{k}}^{e}\right)^{2}+\Delta_{r}^{2}}, \\
\text { with } \quad \xi_{\mathbf{k}}^{e} & =\epsilon_{\mathbf{k}}+e \mu, \quad \epsilon_{\mathbf{k}}=\sqrt{\mathbf{k}^{2}+m^{2}}, \quad e= \pm .
\end{aligned}
$$

Explicit expressions for the matrix elements of the fermion propagator, obtained by inverting Eq. (2), read,

$$
\begin{aligned}
& \mathcal{D}^{\psi \bar{\psi}}\left(i \omega_{n}, \mathbf{k}\right)=\sum_{r} \sum_{e= \pm} \frac{i \omega_{n}-e \xi_{\mathbf{k}}^{e}}{\left(i \omega_{n}\right)^{2}-\left(E_{\mathbf{k} r}^{e}\right)^{2}} \Lambda_{\mathbf{k}}^{-e} \gamma_{0} \mathcal{P}_{r} \\
& \mathcal{D}^{\bar{\psi} \psi}\left(i \omega_{n}, \mathbf{k}\right)=\sum_{r} \sum_{e= \pm} \frac{i \omega_{n}+e \xi_{\mathbf{k}}^{e}}{\left(i \omega_{n}\right)^{2}-\left(E_{\mathbf{k} r}^{e}\right)^{2}} \Lambda_{\mathbf{k}}^{e} \gamma_{0} \mathcal{P}_{r}^{*} \\
& \mathcal{D}^{\psi \psi}\left(i \omega_{n}, \mathbf{k}\right)=-\sum_{r} \sum_{e= \pm} \frac{\Lambda_{\mathbf{k}}^{-e} \gamma_{5}}{\left(i \omega_{n}\right)^{2}-\left(E_{\mathbf{k} r}^{e}\right)^{2}} \mathcal{P}_{r} M \\
& \mathcal{D}^{\bar{\psi} \bar{\psi}}\left(i \omega_{n}, \mathbf{k}\right)=\sum_{r} \sum_{e= \pm} \frac{\Lambda_{\mathbf{k}}^{e} \gamma_{5}}{\left(i \omega_{n}\right)^{2}-\left(E_{\mathbf{k} r}^{e}\right)^{2}} M^{\dagger} \mathcal{P}_{r}
\end{aligned}
$$

The definition and basic properties of the standard energy projectors $\Lambda_{\mathbf{k}}^{e}$ are given in Appendix A 1.

\section{Propagator of the collective modes}

Eqs. (6), (7), and (9) show that the calculation of the inverse propagator, $\Xi$, of the bosonic collective excitations can be split into three independent steps. First, the trace over Dirac indices is always of the form

$$
\operatorname{Tr}_{\mathrm{D}}\left(\Lambda_{\mathbf{k}}^{e} \Lambda_{\mathbf{q}}^{f}\right)=1+e f \frac{m^{2}+\mathbf{k} \cdot \mathbf{q}}{\epsilon_{\mathbf{k}} \epsilon_{\mathbf{q}}}
$$

Second step is the trace over the flavor indices, $\operatorname{Tr}_{\mathrm{F}}$. This will be discussed shortly. Last, the summation over the fermionic Matsubara frequencies $\omega_{n}$ gives rise to functions $I_{r s}^{e f}\left(\mathbf{k}, \mathbf{q} ; i \Omega_{N}\right)$ and $J_{r s}^{e f}\left(\mathbf{k}, \mathbf{q} ; i \Omega_{N}\right)$ defined in Appendix A 2

Putting all the pieces together, the normal and anomalous correlation functions, $\chi^{\Delta \Delta^{*}}$ and $\chi^{\Delta \Delta}$, are given by the formulas

$$
\begin{aligned}
& \chi_{a b}^{\Delta \Delta^{*}}\left(i \Omega_{N}, \mathbf{p}\right)=\frac{1}{G} \delta_{a b}+\frac{1}{2} \sum_{r, s} \operatorname{Tr}_{\mathrm{F}}\left(Q_{a} \mathcal{P}_{r} Q_{b}^{\dagger} \mathcal{P}_{s}^{*}\right) \sum_{e, f} \int \frac{d^{3} \mathbf{k}}{(2 \pi)^{3}} \operatorname{Tr}_{\mathrm{D}}\left(\Lambda_{\mathbf{k}}^{e} \Lambda_{\mathbf{k}-\mathbf{p}}^{f}\right) I_{r s}^{e f}\left(\mathbf{k}, \mathbf{k}-\mathbf{p} ; i \Omega_{N}\right), \\
& \chi_{a b}^{\Delta \Delta}\left(i \Omega_{N}, \mathbf{p}\right)=\frac{1}{2} \sum_{r, s} \operatorname{Tr}_{\mathrm{F}}\left(Q_{a} \mathcal{P}_{r} M Q_{b} \mathcal{P}_{s} M\right) \sum_{e, f} \int \frac{d^{3} \mathbf{k}}{(2 \pi)^{3}} \operatorname{Tr}_{\mathrm{D}}\left(\Lambda_{\mathbf{k}}^{e} \Lambda_{\mathbf{k}-\mathbf{p}}^{f}\right) J_{r s}^{e f}\left(\mathbf{k}, \mathbf{k}-\mathbf{p} ; i \Omega_{N}\right) .
\end{aligned}
$$

In particular, in the normal phase all $\Delta$ 's are equal to zero so that $\chi^{\Delta \Delta}=0$. Moreover, we have $\sum_{r, s} \operatorname{Tr}_{\mathrm{F}}\left(Q_{a} \mathcal{P}_{r} Q_{b}^{\dagger} \mathcal{P}_{s}^{*}\right)=\operatorname{Tr}_{\mathrm{F}}\left(Q_{a} Q_{b}^{\dagger}\right)=N \delta_{a b}$, which can always be enforced by a suitable choice of basis of the flavor algebra. $N$ is a normalization factor which, in principle, can differ for different pairing channels (or, different irreducible representations of the flavor symmetry). In the following, we will assume that the fermions pair in a single channel, but the generalization is obvious and straightforward. After this remark, we can write down the correlation function in the normal phase as a special case of Eq. (10),

$$
\begin{aligned}
& \chi\left(i \Omega_{N}, \mathbf{p}\right)=\frac{1}{G}+\frac{N}{2} \int \frac{d^{3} \mathbf{k}}{(2 \pi)^{3}}\left\{\left[1+\frac{m^{2}+\mathbf{k} \cdot(\mathbf{k}-\mathbf{p})}{\epsilon_{\mathbf{k}} \epsilon_{\mathbf{k}-\mathbf{p}}}\right]\left[\frac{2 f\left(\epsilon_{\mathbf{k}}+\mu\right)-1}{i \Omega_{N}+2 \mu+\epsilon_{\mathbf{k}}+\epsilon_{\mathbf{k}-\mathbf{p}}}+\frac{1-2 f\left(\epsilon_{\mathbf{k}}-\mu\right)}{i \Omega_{N}+2 \mu-\epsilon_{\mathbf{k}}-\epsilon_{\mathbf{k}-\mathbf{p}}}\right]\right. \\
& \left.+2\left[1-\frac{m^{2}+\mathbf{k} \cdot(\mathbf{k}-\mathbf{p})}{\epsilon_{\mathbf{k}} \epsilon_{\mathbf{k}-\mathbf{p}}}\right] \frac{f\left(\epsilon_{\mathbf{k}}+\mu\right)-f\left(\epsilon_{\mathbf{k}-\mathbf{q}}-\mu\right)}{i \Omega_{N}+2 \mu+\epsilon_{\mathbf{k}}-\epsilon_{\mathbf{k}-\mathbf{p}}}\right\},
\end{aligned}
$$


which is equivalent to Eq. (15) in Ref. [36].

Full information about the spectrum of bosonic collective states may be obtained by investigating the spectral density, $\rho(\omega, \mathbf{p})$, associated to the matrix correlation function $\Xi$, defined by

$$
\left[\Xi^{-1}\left(i \Omega_{N}, \mathbf{p}\right)\right]_{a b}=-\int_{-\infty}^{+\infty} \frac{d \omega}{\pi} \frac{\rho_{a b}(\omega, \mathbf{p})}{i \Omega_{N}-\omega} .
$$

In general, we would like to use the relation

$$
\rho_{a b}(\omega, \mathbf{p})=\operatorname{Im}\left[\Xi^{-1}(\omega+i \delta, \mathbf{p})\right]_{a b},
$$

in order to extract the spectral information from the imaginary part of the pair correlation function, analytically continued to the real axis. However, this should be done with some care for Eq. (13) holds only when the spectral density $\rho_{a b}(\omega, \mathbf{p})$ defined by Eq. (12) is real.

\section{Mean-field analysis}

Assuming that in thermodynamic equilibrium the system relaxes to a homogeneous state, the mean-field thermodynamic potential $\Omega_{\mathrm{MF}}$ is given by Eq. (3),

$$
\begin{aligned}
& \frac{\Omega_{\mathrm{MF}}}{V}=\frac{\left|\Delta_{a}\right|^{2}}{G} \\
& -\sum_{r} \sum_{e= \pm} \int \frac{d^{3} \mathbf{k}}{(2 \pi)^{3}}\left[\left(E_{\mathbf{k} r}^{e}-\epsilon_{\mathbf{k}}+2 T \log \left(1+e^{-\beta E_{\mathbf{k} r}^{e}}\right)\right],\right.
\end{aligned}
$$

where we subtracted the energy of the vacuum in order that $\Omega_{\mathrm{MF}}$ expresses the sheer effect of finite temperature and density, and the Cooper pairing.

It should be noted that Eq. (14) embodies two different sets of gap parameters. First, the $\Delta_{a}$ 's, which carry the label of the Cooper pair representation of the symmetry, and represent the order parameters for symmetry breaking. Second, the $\Delta_{r}$ 's, which determine the gaps in the fermion excitation spectrum. While the $\Delta_{a}$ 's transform as an antisymmetric tensor under the symmetry transformations acting on $\psi$ and may in general be complex, the $\Delta_{r}$ 's are by construction invariants of the symmetry and are always real.

These two sets of parameters may be related with the help of the expression $\Delta_{a} \Delta_{b}^{*} Q_{a}^{\dagger} Q_{b}=M M^{\dagger}=\sum_{r} \Delta_{r}^{2} \mathcal{P}_{r}$. By taking the trace we find

$$
N \sum_{a}\left|\Delta_{a}\right|^{2}=\sum_{r} \Delta_{r}^{2}
$$

This clarifies the physical content of the normalization factor $N$ : It counts the number of fermion degrees of freedom participating in the formation of a particular Cooper pair. Plugging this result back into Eq. (14), we immediately arrive at the standard mean-field gap equation and the equation for the particle number density $n$,

$$
\begin{aligned}
\Delta_{r} & =\Delta_{r} N G \sum_{e= \pm} \int \frac{d^{3} \mathbf{k}}{(2 \pi)^{3}} \frac{1-2 f\left(E_{\mathbf{k} r}^{e}\right)}{2 E_{\mathbf{k} r}^{e}}, \\
n & =\sum_{r} \sum_{e= \pm} \int \frac{d^{3} \mathbf{k}}{(2 \pi)^{3}} \frac{e \xi_{\mathbf{k}}^{e}}{E_{\mathbf{k} r}^{e}}\left[1-2 f\left(E_{\mathbf{k} r}^{e}\right)\right] .
\end{aligned}
$$

The total number density $n$ is fixed by the value of the Fermi momentum $k_{\mathrm{F}}$, as $n=k_{\mathrm{F}}^{3} /\left(3 \pi^{2}\right)$ times the number of fermion flavors.

\section{E. Renormalization}

The momentum integral in Eq. (15) (as well as in other equations depending explicitly on the coupling) is badly divergent. This can be taken care of by trading the bare coupling $G$ for the physical $s$-wave scattering length at zero temperature and density, or the renormalized coupling $G_{R}$, defined by [36]

$$
\frac{1}{G}=-\frac{1}{G_{R}}+\frac{N}{2} \int \frac{d^{3} \mathbf{k}}{(2 \pi)^{3}}\left(\frac{1}{\epsilon_{\mathbf{k}}+m}+\frac{1}{\epsilon_{\mathbf{k}}-m}\right) .
$$

As opposed to the case of nonrelativistic fermion matter, the remaining momentum integration is still divergent, but only mildly, logarithmically. In numerical computations, it is regulated with a sharp three-momentum cutoff $\Lambda$. All equations of the theory may be rewritten in such a way that the cancelation of the leading divergences is manifest. The resulting formulas are useful for the numerical implementation, but otherwise are rather cumbersome. Just for illustration, we show here the gap equation and the equation for number density,

$$
\begin{aligned}
& \Delta_{r}=-\frac{1}{2} \Delta_{r} N G_{R} \sum_{e= \pm} \int \frac{d^{3} \mathbf{k}}{(2 \pi)^{3}}\left[-\frac{m^{2}}{\mathbf{k}^{2} \epsilon_{\mathbf{k}}}-\frac{2 f\left(E_{\mathbf{k} r}^{e}\right)}{E_{\mathbf{k} r}^{e}}\right. \\
&\left.+\frac{2 \mu^{2}}{E_{\mathbf{k} r}^{e} E_{\mathbf{k} r}^{-e}\left(E_{\mathbf{k} r}^{e}+E_{\mathbf{k} r}^{-e}\right)}-\frac{\Delta_{r}^{2}}{\epsilon_{\mathbf{k}}} \frac{1}{E_{\mathbf{k} r}^{e}\left(E_{\mathbf{k} r}^{e}+\xi_{\mathbf{k}}^{e}\right)}\right] \\
& n=\sum_{r} \sum_{e= \pm} \int \frac{d^{3} \mathbf{k}}{(2 \pi)^{3}}\left[-2 e \frac{\xi_{\mathbf{k}}^{e}}{E_{\mathbf{k} r}^{e}} f\left(E_{\mathbf{k} r}^{e}\right)\right. \\
&\left.\quad+\frac{\mu \Delta_{r}^{2}}{E_{\mathbf{k} r}^{e} E_{\mathbf{k} r}^{-e}\left(E_{\mathbf{k} r}^{e}+E_{\mathbf{k} r}^{-e}\right)}\left(1+\frac{E_{\mathbf{k} r}^{e}+\xi_{\mathbf{k}}^{e}}{E_{\mathbf{k} r}^{-e}+\xi_{\mathbf{k}}^{-e}}\right)\right]
\end{aligned}
$$

The procedure outlined above is sufficient to renormalize the mean-field thermodynamic potential and its derivates, the gap and number equations. On the other hand, one must be more careful when calculating the correlator $\Xi_{a b}(5)$, or in general any fermion loop with nonzero external momentum. The point is that the momentum assignment for the internal fermion lines is not completely fixed by momentum conservation in the interaction vertices; it is unique up to a shift of the integration variable. Such a shift, however, is not permitted 
in an integral with stronger than logarithmic divergence [50]. Therefore, we are led to consider the momentum assignment to the fermion propagators in Eqs. (6) and (77) as a part of the definition of the model. As already explained above, the integrals are then evaluated with a sharp cutoff on the three-momentum $\mathbf{k}$.

Let us finally remark that one might at first sight think it would be natural to impose the cutoff directly on the arguments of the fermion propagators in the loop, i.e., on both $\mathbf{k}$ and $\mathbf{k}-\mathbf{p}$ in Eqs. (6) and (7); this would roughly correspond to discretizing the coordinate space. Nevertheless, this would destroy the expected low-energy dynamics of our model, based on the Nambu-Goldstone (NG) modes of the spontaneously broken symmetry the inverse propagator (6) would develop a linearly divergent piece, proportional to $|\mathbf{p}|$.

\section{TWO-FLAVOR COLOR SUPERCONDUCTOR}

Now we apply the general formulas derived in the previous section to the particular model studied by Abuki [36]. It describes color superconductivity in quark matter consisting of two quark flavors, which have for simplicity equal masses and chemical potentials. The field $\psi$ now carries the index of the fundamental representation of the symmetry group $\mathrm{SU}(3) \times \mathrm{SU}(2)$. Since the weak-coupling studies of quark matter indicate attraction between two quarks in the color-antitriplet channel, we assume that the matrices $Q_{a}$ have the structure $\left(Q_{a}\right)_{b c}^{i j}=\epsilon^{i j} \epsilon_{a b c}$, where $i, j$ from now on denote the flavor $\mathrm{SU}(2)$ indices and $a, b, c$ the color $\mathrm{SU}(3)$ ones. The order parameter $\Delta_{a}$ thus transforms as an $\mathrm{SU}(2)$-singlet and an SU(3)-antitriplet. As usual it is chosen to be real and to point in the third (anti-blue) direction in the color space. In the following, we write simply $\Delta$ instead of $\Delta_{3}$; this will help us distinguish the constant order parameter from the fluctuation fields without having to introduce further notation.

For this particular symmetry structure and the choice of vacuum, we find $N=4$ and $M M^{\dagger}=\Delta^{2} \operatorname{diag}(1,1,0)$ in the color space (in the flavor space, it is simply the unit matrix). So in this case, the projectors $\mathcal{P}_{r}$ simply project on the individual colors. The red and green quarks, participating in the pairing, are gapped, while the blue quarks remain gapless. As is well known, the global $\mathrm{SU}(3) \times \mathrm{U}(1)$ symmetry of the bosonized action (3) is broken by the Cooper pair condensate $\Delta$ down to $\mathrm{SU}(2) \times \mathrm{U}(1)_{\mathrm{Q}}$, operating exclusively on $\Delta_{1}$ and $\Delta_{2}$ [51]. The unbroken symmetry implies degeneracy between $\Delta_{1}$ and $\Delta_{2}$. The pair correlation matrix $\Xi_{a b}$ is then diagonal and $\Xi_{11}=\Xi_{22}$. Moreover, $\Xi_{11}$ has only the normal part, thanks to the existence of the unbroken $\mathrm{U}(1)_{\mathrm{Q}}$ charge. Evaluating explicitly the color-flavor traces in Eqs. (10) and (11), we obtain the expressions

$$
\begin{aligned}
& \chi_{11}^{\Delta \Delta^{*}}\left(i \Omega_{N}, \mathbf{p}\right)=\frac{1}{G}+\sum_{e, f} \int \frac{d^{3} \mathbf{k}}{(2 \pi)^{3}}\left[1+e f \frac{m^{2}+\mathbf{k} \cdot(\mathbf{k}-\mathbf{p})}{\epsilon_{\mathbf{k}} \epsilon_{\mathbf{k}-\mathbf{p}}}\right]\left[I_{23}^{e f}\left(\mathbf{k}, \mathbf{k}-\mathbf{p} ; i \Omega_{N}\right)+I_{32}^{e f}\left(\mathbf{k}, \mathbf{k}-\mathbf{p} ; i \Omega_{N}\right)\right], \\
& \chi_{33}^{\Delta \Delta^{*}}\left(i \Omega_{N}, \mathbf{p}\right)=\frac{1}{G}+2 \sum_{e, f} \int \frac{d^{3} \mathbf{k}}{(2 \pi)^{3}}\left[1+e f \frac{m^{2}+\mathbf{k} \cdot(\mathbf{k}-\mathbf{p})}{\epsilon_{\mathbf{k}} \epsilon_{\mathbf{k}-\mathbf{p}}}\right] I_{12}^{e f}\left(\mathbf{k}, \mathbf{k}-\mathbf{p} ; i \Omega_{N}\right), \\
& \chi_{33}^{\Delta \Delta}\left(i \Omega_{N}, \mathbf{p}\right)=-2 \Delta^{2} \sum_{e, f} \int \frac{d^{3} \mathbf{k}}{(2 \pi)^{3}}\left[1+e f \frac{m^{2}+\mathbf{k} \cdot(\mathbf{k}-\mathbf{p})}{\epsilon_{\mathbf{k}} \epsilon_{\mathbf{k}-\mathbf{p}}}\right] J_{12}^{e f}\left(\mathbf{k}, \mathbf{k}-\mathbf{p} ; i \Omega_{N}\right) .
\end{aligned}
$$

Note that the quark loop contributing to $\Xi_{33}$ is symmetric: Both propagators correspond to gapped quarks. On the other hand, the loop in $\Xi_{11}$ consists of one gapped and one ungapped propagator and the general formula (10) requires it to be symmetrized.

\section{A. Nambu-Goldstone bosons}

Spontaneous symmetry breaking leads to the existence of NG bosons, soft fluctuations of the order parameter. Vacuum expectation value of a color antitriplet breaks five generators of the color $\mathrm{SU}(3)$ so that we should expect five NG bosons. However, in our model where the color symmetry is just global unlike in quantum chromo- dynamics, the Noether charge corresponding to the GellMann matrix $\lambda_{8}$ acquires nonzero density. This implies that there are only three NG bosons, two of which have quadratic dispersion law at low momentum, being the so-called type-II NG bosons [40, 41, 42]. These couple to the two pairs of generators, $\left(\lambda_{4}, \lambda_{5}\right)$ and $\left(\lambda_{6}, \lambda_{7}\right)$, whose commutator contains a $\lambda_{8}$ piece and hence has nonzero density. Therefore, they will be found in the spectrum of excitations of $\Delta_{1}$ and $\Delta_{2}$. The remaining, type-I NG boson couples to the spontaneously broken linear combination of $\lambda_{3}$ and $\lambda_{8}$ and will be found as an excitation of $\Delta_{3}$.

At zero temperature, the presence of NG modes in our model may easily be demonstrated analytically. We set $\mathbf{p}=\mathbf{0}$, use the gap equation (15), and analytically con- 
tinue the pair correlation functions to real frequency (or, more precisely, to the vicinity of the real axis),

$$
\begin{aligned}
& \chi_{11}^{\Delta \Delta^{*}}(\omega, \mathbf{0})=2 \omega \int \frac{d^{3} \mathbf{k}}{(2 \pi)^{3}}\left\{\frac{1}{E_{\mathbf{k}}^{+}} \frac{1}{\omega+E_{\mathbf{k}}^{+}+\xi_{\mathbf{k}}^{+}}\right. \\
&\left.+\frac{1}{E_{\mathbf{k}}^{-}}\left[\frac{\theta\left(\xi_{\mathbf{k}}^{-}\right)}{\omega-E_{\mathbf{k}}^{-}-\left|\xi_{\mathbf{k}}^{-}\right|}+\frac{\theta\left(-\xi_{\mathbf{k}}^{-}\right)}{\omega+E_{\mathbf{k}}^{-}+\left|\xi_{\mathbf{k}}^{-}\right|}\right]\right\}, \\
& \chi_{33}^{\Delta \Delta^{*}}(\omega, \mathbf{0})=2 \sum_{e= \pm} \int \frac{d^{3} \mathbf{k}}{(2 \pi)^{3}} \frac{1}{E_{\mathbf{k}}^{e}} \frac{\omega^{2}-2 \Delta^{2}-2 e \omega \xi_{\mathbf{k}}^{e}}{\omega^{2}-\left(2 E_{\mathbf{k}}^{e}\right)^{2}} \\
& \chi_{33}^{\Delta \Delta}(\omega, \mathbf{0})=4 \Delta^{2} \sum_{e= \pm} \int \frac{d^{3} \mathbf{k}}{(2 \pi)^{3}} \frac{1}{E_{\mathbf{k}}^{e}} \frac{1}{\omega^{2}-\left(2 E_{\mathbf{k}}^{e}\right)^{2}} .
\end{aligned}
$$

[We have dropped the index $r$ so that $E_{\mathbf{k}}^{e}$ is now defined by Eq. (8) with the gap $\Delta$ inserted.] We can immediately see that $\chi_{11}^{\Delta \Delta^{*}}(\omega, \mathbf{0}) \rightarrow 0$ as $\omega \rightarrow 0$, which proves the existence of $\mathrm{NG}$ excitations coupled to $\Delta_{1}$ and $\Delta_{2}$. These are therefore interpreted as massless bound states of one gapped and one gapless quark (green and blue, and red and blue, respectively).

The correlation functions of $\Delta_{3}$ do not go to zero at vanishing frequency. However, here the propagator is given by inverting the matrix $\Xi$ (5). Since in the limit $\omega \rightarrow 0$ we have

$$
\chi_{33}^{\Delta \Delta^{*}}(0, \mathbf{0})=-\chi_{33}^{\Delta \Delta}(0, \mathbf{0}),
$$

the determinant of $\Xi$ will be zero and the existence of a NG mode is thus proved.

At zero temperature, we can of course go beyond the mere demonstration of the existence of NG bosons, and determine their low-momentum dispersion relation. This may be done in the standard manner, by expanding the correlation functions in powers of momentum. Let us just remark that at nonzero temperature but still in the superfluid phase, there are kinematical regions where the sharp NG peak in the pair correlation spectrum is obscured by Landau damping - the emission or absorption of the NG boson by a quark. In such regions the momentum expansion of the correlation functions is not possible [43].

Here we concentrate on the doublet of type-II NG bosons coupled to $\Delta_{1,2}$, aiming to investigate the effect of the expected quadratic onset of their dispersion relation. At low frequency and momentum, the inverse propagator $\chi_{11}^{\Delta \Delta^{*}}$ is expanded as

$$
\chi_{11}^{\Delta \Delta^{*}}(\omega, \mathbf{p})=-a \omega+b \mathbf{p}^{2} .
$$

The coefficients $a, b$ determine the low-momentum dispersion relation of the NG boson by $\omega(\mathbf{p})=\mathbf{p}^{2} b / a$. They are given by the explicit expressions

$$
a=\frac{2}{\Delta^{2}} \sum_{e= \pm} \int \frac{d^{3} \mathbf{k}}{(2 \pi)^{3}}\left[\frac{e \xi_{\mathbf{k}}^{e}}{E_{\mathbf{k}}^{e}}-\operatorname{sgn}\left(e \xi_{\mathbf{k}}^{e}\right)\right]
$$

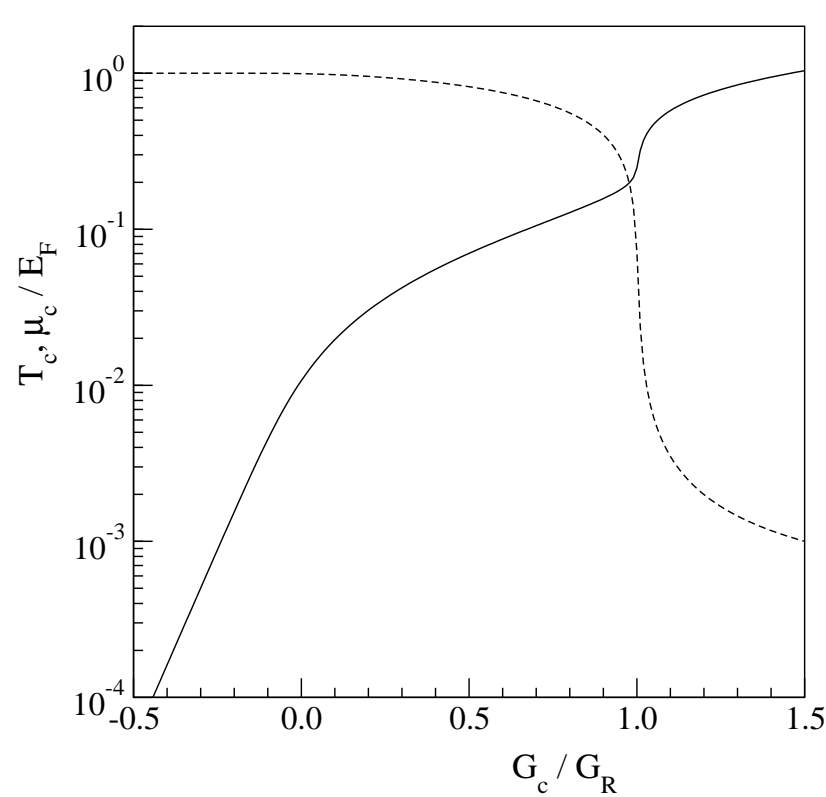

FIG. 1: The critical temperature (solid) and chemical potential (dashed) in units of the Fermi energy, as a function of the inverse coupling. Three different regimes are distinguished: $\operatorname{BCS}\left(G_{c} / G_{R} \lesssim 0\right)$, BEC $\left(0 \lesssim G_{c} / G_{R} \lesssim 1\right)$, and RBEC $\left(G_{c} / G_{R} \gtrsim 1\right)$.

$$
\begin{aligned}
& b= \theta(\mu-m) \frac{\left(\mu^{2}-m^{2}\right)^{3 / 2}}{3 \pi^{2} \Delta^{2} \mu}+\sum_{e= \pm} \int \frac{d^{3} \mathbf{k}}{(2 \pi)^{3}} \\
& \times\left[\left(1-\frac{\mathbf{k}^{2}}{3 \epsilon_{\mathbf{k}}^{2}}\right) \frac{1}{\epsilon_{\mathbf{k}} E_{\mathbf{k}}^{e}}\left(\frac{\operatorname{sgn} \xi_{\mathbf{k}}^{-e}}{E_{\mathbf{k}}^{e}+\left|\xi_{\mathbf{k}}^{-e}\right|}+\frac{\xi_{\mathbf{k}}^{e}}{2\left(E_{\mathbf{k}}^{e}\right)^{2}}\right)\right. \\
&\left.\quad-\frac{2 \mathbf{k}^{2}}{3 \epsilon_{\mathbf{k}}^{2}} \frac{1}{E_{\mathbf{k}}^{e}} \frac{1}{\left(E_{\mathbf{k}}^{e}+\left|\xi_{\mathbf{k}}^{e}\right|\right)^{2}}+\frac{\mathbf{k}^{2}}{2 \epsilon_{\mathbf{k}}^{2}} \frac{\Delta^{2}}{\left(E_{\mathbf{k}}^{e}\right)^{5}}\right]
\end{aligned}
$$

Note that $a$ is actually equal to $1 / \Delta^{2}$ times the difference of the density of a gapped and a gapless color [cf. Eq. (16), the factor 2 arises from the existence of two quark flavors for each color]. This in turn is proportional to the density of $\lambda_{8}$ as it should, for it is this non-Abelian charge which, according to general theorems [42, 44] gives rise to type-II NG bosons.

\section{B. Numerical results}

In this section, we present numerical results that illustrate the general conclusions made above. In order to achieve concrete numbers, we use the same set of parameters as Abuki [36] so that our results in the superfluid phase can be matched to his for the normal phase. In particular, we choose the Fermi momentum $k_{\mathrm{F}}=0.2 \mathrm{~m}$ and a cutoff to regulate the logarithmic-divergent integrals as $\Lambda=5 \mathrm{~m}$. The renormalized coupling $G_{R}$ is represented in units of the critical coupling $G_{c}$, at which the mass of the diquark molecule in vacuum goes to zero. This signifies the instability of the vacuum itself with respect to pair formation, and the onset of relativistic 


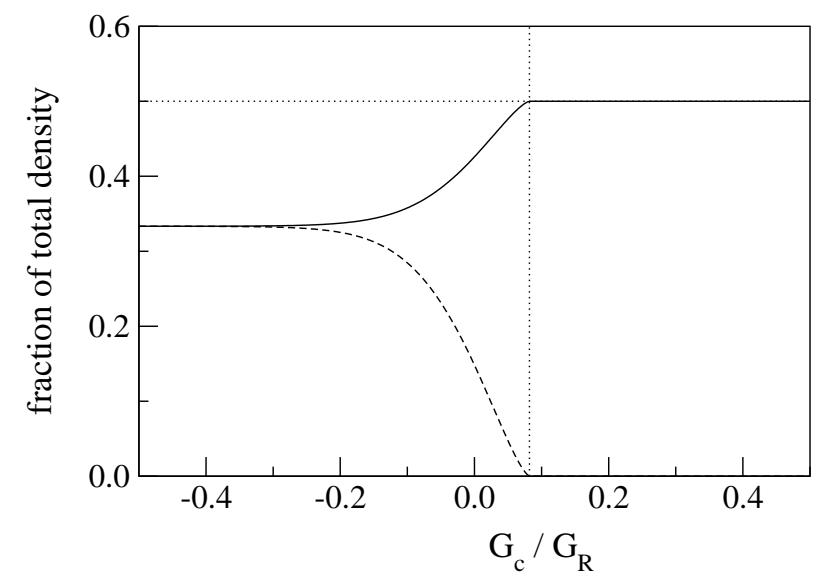

FIG. 2: Zero-temperature density of quarks of a single paired (solid) and unpaired (dashed) color at zero temperature, expressed as a fraction of the fixed total density. Recall that there are two paired colors (red and green) and one unpaired one (blue).

Bose-Einstein condensation (RBEC).

\section{Phase diagram}

The dependence of the critical temperature on the renormalized coupling is displayed in Fig. 1. It is instructive to compare this mean-field plot to a similar one achieved by Abuki and Nishida [35, 36], who included the effects of Gaussian fluctuations. (To be precise, they combine the mean-field Thouless criterion [45] with the equation for particle number that includes the contribution of bosonic quasiparticles.) It is clearly seen that as expected, the mean-field approximation is reliable in the BCS regime (negative coupling), while it fails by about an order of magnitude on the BEC side. The reason is, of course, that the mean-field thermodynamic potential includes only the contribution of the fermions, whereas in the $\mathrm{BEC}$ regime the total number density is dominated by the bosons. In the RBEC limit (that is for $G_{c} / G_{R} \gtrsim 1$ ), both bosons and fermions are excited significantly, and the reliability of the mean-field approximation again improves.

In Fig. 2 we show the contributions of the paired and unpaired quarks to the total density at zero temperature. In the BCS limit the unpaired blue quarks form a sharp Fermi sea, while the paired red and green quarks occupy a Fermi sea, smeared about the Fermi surface at the scale of the gap. However, at $G_{c} / G_{R} \approx 0.08$ the chemical potential decreases below the fermion mass and the Fermi sea can no longer exist. This marks the onset of the BEC regime. From this point on, the whole density at zero temperature is provided by the condensate of the bound diquark molecules, made of red and green quarks. The blue quarks can only be excited thermally.

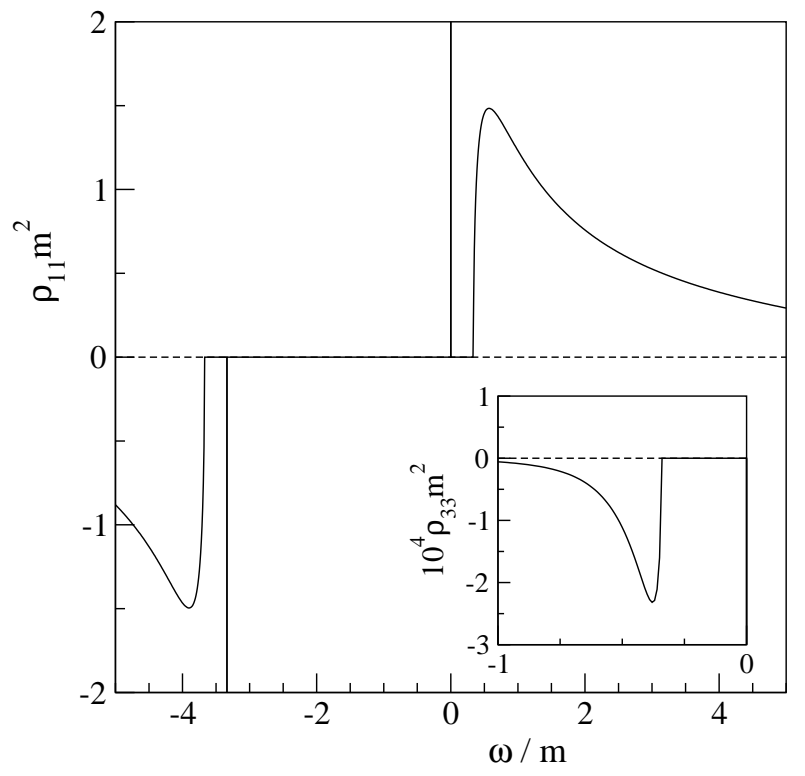

FIG. 3: Normal component of the spectral density matrix at zero temperature and momentum for $G_{c} / G_{R}=0.5$. The large-scale plot applies equally well to $\rho_{11}$ and $\rho_{33}$. In the inset we show the tiny contribution of the particle-particle continuum at negative frequencies to $\rho_{33}$, which is absent in $\rho_{11}$.

\section{Bosonic spectrum at zero temperature and momentum}

As already remarked before, the correlation function $\Xi_{a b}$ is diagonal in the color-flavor space, owing to the choice of the order parameter $\Delta$ to point in the third direction in the anticolor space. Moreover, since we choose $\Delta$ real, the spectral density $\rho_{a b}$ defined by Eq. (12) is real and we can use Eq. (13) to calculate it.

In the normal phase, the matrix $\Xi$ is simply proportional to the unit matrix and there is a single spectral density, which has been calculated at the critical temperature by Abuki [36]. In the superfluid phase, we have one spectral density for $a=b=1,2$ and a symmetric $2 \times 2$ matrix for $a=b=3$. In Fig. 3 we give a sample numerical calculation of the normal parts of these spectral densities for $G_{c} / G_{R}=0.5$.

Several comments are in order here. First, in the normal phase an isolated bound state (corresponding to a diquark or antidiquark molecule) exists only on the BEC side of the crossover. In the superfluid phase, the NG bound state is present for all values of the coupling, as guaranteed by the Goldstone theorem. On the other hand, the antiboson pole appears only on the BEC side of the crossover, just as in the normal phase. It should be stressed that the positions of the antiboson poles in $\rho_{11}$ and $\rho_{33}$ differ, simply because one of the bosons is composed of a gapped and an ungapped (anti)quark, while the other one of two gapped (anti)quarks. Indeed, the pole in $\rho_{33}$ occurs at a smaller (negative) $\omega$; the corresponding boson is heavier. Nevertheless, for 


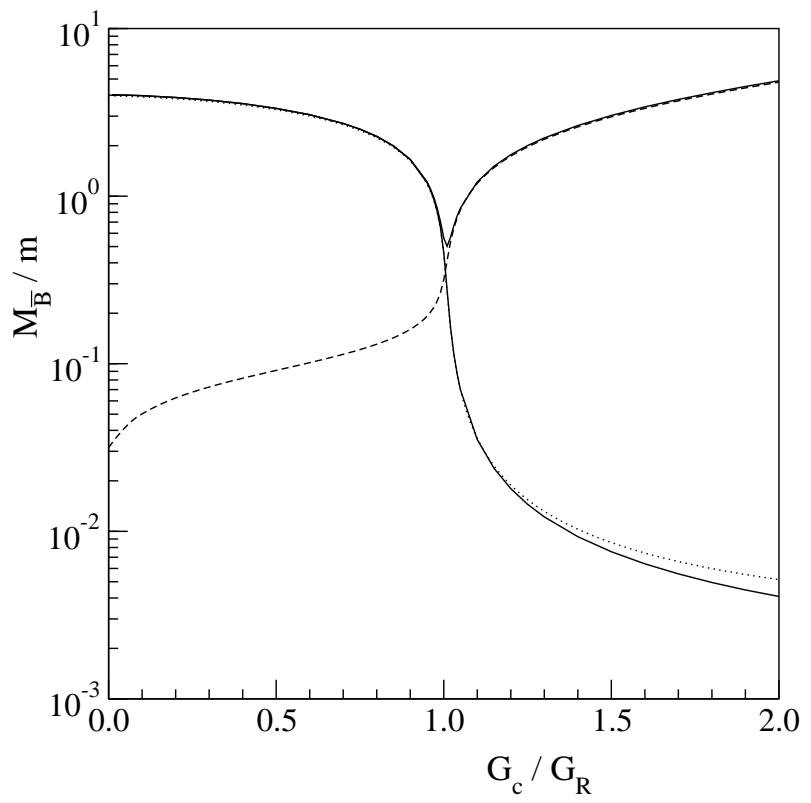

FIG. 4: Absolute value of the position of the antiboson pole in $\rho_{11}$ (lower solid line) and $\rho_{33}$ (upper solid line) at zero temperature and momentum. For comparison, we also show the values of $2 \Delta$ (dashed) and $4 \mu$ (dotted).

$G_{c} / G_{R}=0.5$ the difference is so small that it cannot be seen in Fig. 3 ,

Apart from the presence of the isolated antiboson pole on the BEC side of the crossover (and its absence on the BCS side), the qualitative appearance of the spectrum does not change as the coupling is varied. As noted above, the antiboson pole has different positions in $\rho_{11}$ and $\rho_{33}$. The same, of course, holds for the onset of the continuum in these spectra. As we proceed from the BCS to the $\mathrm{BEC}$ to the RBEC regime, the gap in the spectrum of red and green quarks increases, and the difference between $\rho_{11}$ and $\rho_{33}$ becomes more pronounced.

In Fig. 4 we show the coupling-dependence of the two antiboson masses. In the BEC regime, they are almost equal and essentially determined by the chemical potential. However, this changes dramatically in the RBEC regime. While the pole in $\rho_{33}$ appears near the two-antiparticle continuum, now dominated by the large value of the gap, the antiboson pole in $\rho_{11}$ is still driven by the chemical potential. We checked numerically that as the temperature is increased, the mass of the antiboson in $\rho_{33}$ follows (twice) the value of the gap until very close to the phase transition, where it merges with the mass of the antiboson in $\rho_{11}$. This is in agreement with Ref. [36] where it was shown that in the normal phase, both masses (being equal) are determined by the chemical potential.

Another fact to note is the order-of-magnitude suppression of the anomalous spectral density $\rho_{33}^{\Delta \Delta}$. This is due to the fact that, with the exception of the RBEC limit, the gap is much smaller than other scales in the theory such as the Fermi energy or chemical potential. This tiny off-diagonal (in the Nambu space) element of the correlation function matrix $\Xi$ then induces mixing between the bound boson and antiboson states, or in other words, breaks the conservation of the baryon number carried by the quarks. Consequently, the spectral density $\rho_{33}^{\Delta \Delta^{*}}$ displays, besides the expected two-particle continuum at positive frequency, also an analogous continuum at negative frequency, yet with much smaller spectral weight. In $\rho_{11}^{\Delta \Delta^{*}}$, this continuum is absent due to the existence of the conserved charge of the unbroken $\mathrm{U}(1)_{\mathrm{Q}}$.

\section{Bosonic spectrum at nonzero temperature and momentum}

At nonzero temperature and momentum the spectrum becomes more rich. The bound-state peaks acquire nonzero (even though tiny) width and the Landau damping appears. Having in mind that the low-energy dynamics of the system in the superfluid phase is dominated by the NG bosons, we display in Fig. 5 the positivefrequency parts of the spectral density $\rho_{11}$; each panel collects the spectra for several values of the momentum. The NG excitations are manifested by isolated peaks below the two-particle continuum. Comparison of spectra for temperatures close to zero and to the phase transition reveals the temperature effects: The overall increase of the background due to thermal excitations, and the Landau damping, especially strong in the RBEC regime. We can also see how with increasing momentum the NG boson peaks get closer to the continuum; at a certain point they will reach the continuum and disappear from the spectrum as isolated poles.

\section{NG boson dispersion relations}

In addition to the full spectra, we also studied the dispersion relations of the NG bosons at zero temperature. These can be extracted from the spectral densities and, in case of the type-II NG bosons, compared to the analytical low-momentum prediction from Eq. (17). The result is shown in Fig. 6 .

General consequences of spontaneous symmetry breaking in presence of nonzero charge density [42] require that the dispersion relation of the NG boson in $\rho_{11}$ and $\rho_{33}$ is, at low momentum, quadratic and linear, respectively. The scale that defines this low-momentum region is typically set by the symmetry breaking. Above this scale, the NG boson dispersion relation is no longer fixed by the general properties of symmetry breaking, but rather is determined by the detailed dynamics of the system under consideration. In some cases, the NG boson may even cease to exist at high enough momentum; in our model, this is reflected by its disappearance in the continuum.

We display the dispersion relations in a logarithmic scale in order to exhibit their power-law behavior. Apparently, the low-momentum region governed by the 

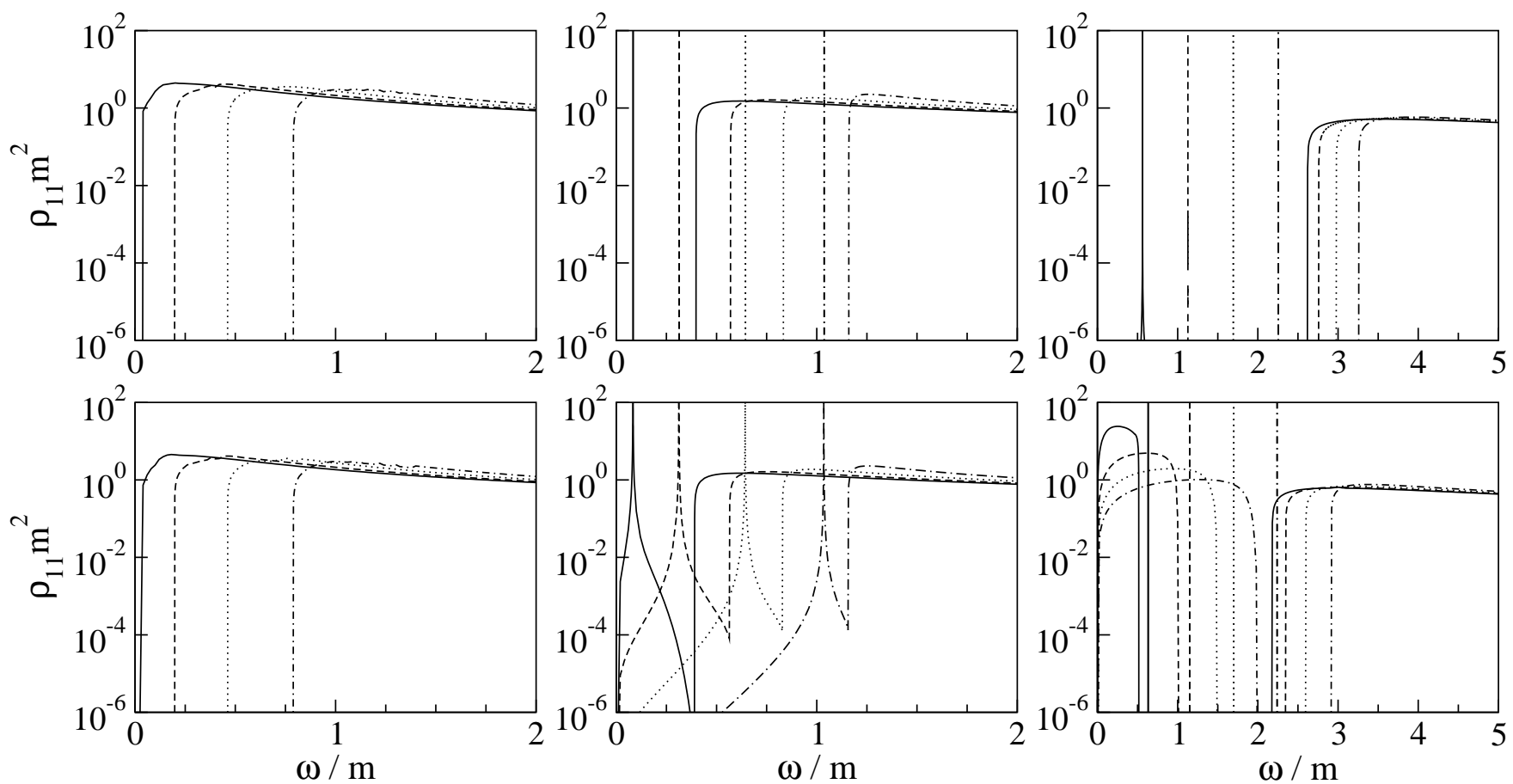

FIG. 5: Positive-frequency part of the $\rho_{11}$ spectral density at nonzero temperature and momentum. First column: BCS regime, $G_{c} / G_{R}=-0.35$; second column: BEC regime, $G_{c} / G_{R}=0.5$; third column: RBEC regime, $G_{c} / G_{R}=1.35$. The upper line shows spectra at low temperature, $T=0.05 T_{c}$, the lower line near the phase transition, $T=0.95 T_{c}$. The four curves in each spectrum correspond respectively to $|\mathbf{p}|=0.5 \mathrm{~m}$ (solid), $|\mathbf{p}|=1.0 \mathrm{~m}$ (dashed), $|\mathbf{p}|=1.5 \mathrm{~m}$ (dotted), and $|\mathbf{p}|=2.0 \mathrm{~m}$ (dash-dotted).
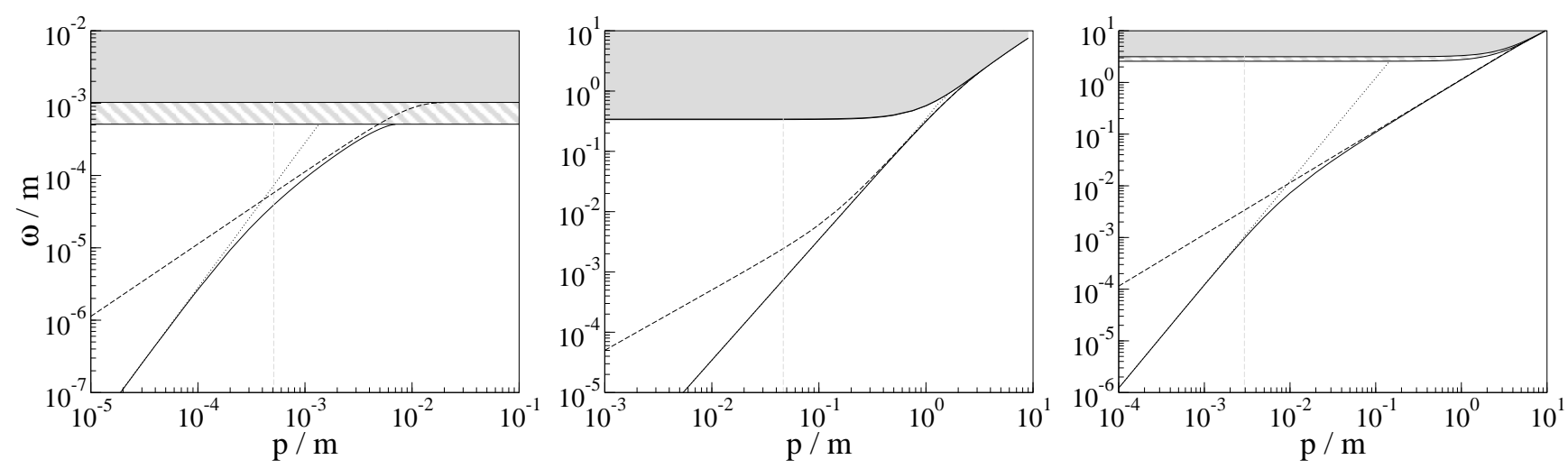

FIG. 6: Dispersion relations of the NG bosons. First plot: BCS regime, $G_{c} / G_{R}=-0.35$; second plot: BEC regime, $G_{c} / G_{R}=$ 0.5; third plot: RBEC regime, $G_{c} / G_{R}=1.35$. In all graphs, the dispersion relation of the type-II NG boson in $\rho_{11}$ (solid), its low-momentum prediction (dotted), as well as the dispersion relation of the type-I NG boson in $\rho_{33}$ (dashed) are shown. The hatched-gray and solid-gray regions denote the continuum in $\rho_{11}$ and $\rho_{33}$, respectively. The dashed gray line indicates the value of the gap (BCS, BEC), or the chemical potential (RBEC).

broken symmetry is indeed defined by the symmetrybreaking scale, i.e., the gap. The only exception is the RBEC limit where the predicted quadratic dispersion of the type-II NG boson is observed only below the scale of the chemical potential, which is much smaller than the gap. This is because above the chemical potential the particles and antiparticles become nearly degenerate and all effects of finite density are strongly suppressed.

In the BEC regime, we can even distinguish three regions of momentum with physically distinct behavior. First, at $|\mathbf{p}| \lesssim \Delta$, the dispersions of the NG bosons are governed by the broken symmetry and match the classification into type I and type II. Second, for momenta larger than a few times the gap but smaller than the 


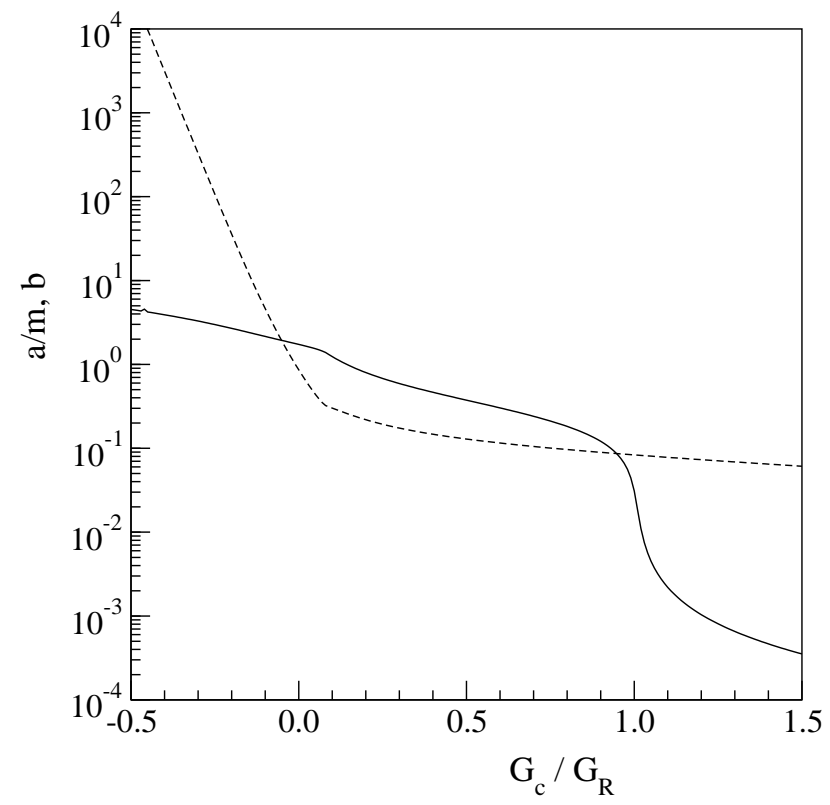

FIG. 7: Coefficients of the type-II NG boson dispersion relation at zero temperature. $a / m$ is plotted by the solid line and $b$ by the dashed one.

fermion mass $m$, the dispersions of all NG bosons become quadratic. This is in accord with the Bogolyubov theory of nonrelativistic BEC [46]. Finally, for $|\mathbf{p}| \gtrsim m$ we enter the relativistic regime and the dispersions become linear again. (Although it cannot be seen in Fig. 6, the NG bosons disappear in the continuum at $|\mathbf{p}| \approx 3.6 \mathrm{~m}$. So in a linear scale, this relativistic domain actually covers a large part of the dispersion relations.)

Let us also remark that in the BCS regime, the frequency marking the onset of the two-particle continuum remains strictly constant for all values of momentum displayed in Fig. 6. The reason is that only in the BCS regime has the dispersion of the fermionic quasiparticles a nontrivial minimum. Consequently, for all momenta $|\mathbf{p}|$ up to the Fermi momentum, $k_{F}$, the pair mode may decay into two fermions both lying on their Fermi surfaces. So the continuum sets at $\omega=\Delta$ in $\rho_{11}$ and at $\omega=2 \Delta$ in $\rho_{33}$.

Another hint on the range of momentum where the universal predictions of the broken symmetry apply is provided by the size of the coefficients $a, b$ defined by Eq. (17). In Fig. 7 we plot these coefficients against the inverse coupling. Apparently, the coefficient $a$ which gives rise to the quadratic onset of the dispersion of the typeII NG boson, is suppressed in both the BCS and RBEC regimes. In the BCS limit, this is because the populations of the gapped and ungapped quarks are almost the same and hence the density of the non-Abelian charge $\lambda_{8}$ goes to zero. In the RBEC limit, the reason is the above-mentioned particle-antiparticle symmetry.

\section{CONCLUSION}

In this paper we studied the relativistic BCS-BEC crossover in a class of NJL-type models. Within the mean-field approximation, we investigated the superfluid phase with particular focus on the collective bosonic excitations. The formalism we developed is general enough to cover all possible spin-zero pairing patterns. The calculation of the thermodynamic potential up to one bosonic loop splits into a kinematic and an algebraic part. The kinematic part is universal and the choice of the pairing pattern enters only through the values of the gap parameters in the fermionic quasiparticle dispersion relations. The algebraic part bears all necessary information about the pairing pattern and can be done at once by means of a simple trace.

Despite the apparent generality of our model Lagrangian (11), two extensions are necessary before the model can claim phenomenological relevance for relativistic BCS-BEC crossover. First, it would be desirable to have different chemical potentials for the individual fermion flavors in order to be able to account for external (e.g. magnetic) fields, or for the requirement of overall charge neutrality. Second, we should also include fermion-antifermion pairing channels so that we can describe the constituent quark masses and the competition between the chiral and diquark condensates.

As a particular application, we described the BCSBEC crossover in the two-flavor color superconductor. We investigated in detail the spectrum of NG bosons. Their low-momentum behavior was checked to comply with the general consequences of spontaneous symmetry breaking at finite charge density. Concretely, the five broken-symmetry generators correspond to one NG boson with a linear dispersion relation, and a doublet of NG bosons with a quadratic dispersion relation. This agrees with the general counting rules for the NG bosons in Lorentz-noninvariant systems [40, 42, 47].

At the very end it should be emphasized once again that the mean-field approximation itself is not sufficient to describe the crossover accurately. However, the investigation of the spectrum of bosonic collective states that we performed here, is to be understood as a necessary first step towards its systematic improvement.

\section{Acknowledgments}

The author is indebted to H. Abuki, J. O. Andersen, J. Hošek, D. H. Rischke, and A. Sedrakian for fruitful discussions and/or critical reading of the manuscript. He is also grateful to P. Kolorenč and D. Parganlija for technical help on computing issues. The present work was in part supported by a Research Fellowship from the Alexander von Humboldt Foundation, and by the GA CR grant No. 202/06/0734. 


\section{APPENDIX A: SOME USEFUL FORMULAS}

\section{Energy projectors}

We use the standard energy projectors $\Lambda_{\mathbf{k}}^{e}$ [39], associated to the solutions of the Dirac equation for a free fermion of mass $m$, with energy $e \epsilon_{\mathbf{k}}, e= \pm$,

$$
\Lambda_{\mathbf{k}}^{e}=\frac{1}{2}\left[1+\frac{e}{\epsilon_{\mathbf{k}}} \gamma_{0}(\boldsymbol{\gamma} \cdot \mathbf{k}+m)\right]
$$

We use the following properties of the projectors,

$$
\begin{gathered}
\not k \pm \mu \gamma_{0}-m=\gamma_{0} \sum_{e= \pm}\left(k_{0} \pm \mu-e \epsilon_{\mathbf{k}}\right) \Lambda_{\mathbf{k}}^{e}, \\
\gamma_{0} \Lambda_{\mathbf{k}}^{e} \gamma_{0}=\Lambda_{-\mathbf{k}}^{e}, \quad \gamma_{5} \Lambda_{\mathbf{k}}^{e} \gamma_{5}=\Lambda_{-\mathbf{k}}^{-e} .
\end{gathered}
$$

\section{Matsubara sums}

Computation of the fermion loop contribution to the inverse boson propagator, involves the following two types of fermionic Matsubara sums [48],

$$
\begin{aligned}
& T \sum_{n} \frac{i \omega_{n}-a}{\left(i \omega_{n}\right)^{2}-A^{2}} \frac{i\left(\omega_{n}-\Omega_{N}\right)+b}{\left(i \omega_{n}-i \Omega_{N}\right)^{2}-B^{2}} \\
& \quad=-\frac{1}{4 A B} \sum_{e_{1}, e_{2}} \frac{\left(A+e_{1} a\right)\left(B+e_{2} b\right)}{i \Omega_{N}+e_{1} A+e_{2} B} \frac{f\left(e_{1} A\right) f\left(e_{2} B\right)}{n\left(e_{1} A+e_{2} B\right)},
\end{aligned}
$$

$$
\begin{aligned}
T \sum_{n} & \frac{1}{\left(i \omega_{n}\right)^{2}-A^{2}} \frac{1}{\left(i \omega_{n}-i \Omega_{N}\right)^{2}-B^{2}} \\
& =\frac{1}{4 A B} \sum_{e_{1}, e_{2}} \frac{e_{1} e_{2}}{i \Omega_{N}+e_{1} A+e_{2} B} \frac{f\left(e_{1} A\right) f\left(e_{2} B\right)}{n\left(e_{1} A+e_{2} B\right)}
\end{aligned}
$$

where $f(x)=1 /\left(e^{\beta x}+1\right)$ and $n(x)=1 /\left(e^{\beta x}-1\right)$ are the Fermi and Bose distributions, respectively, and $\Omega_{N}$ is an arbitrary (external) bosonic Matsubara frequency.

In the main text, we use these two respective formulas with $a=e \xi_{\mathbf{k}}^{e}, b=f \xi_{\mathbf{q}}^{f}, A=E_{\mathbf{k} r}^{e}$, and $B=E_{\mathbf{q} s}^{f}$ to define the quantities $I_{r s}^{e f}\left(\mathbf{k}, \mathbf{q} ; i \Omega_{N}\right)$ and $J_{r s}^{e f}\left(\mathbf{k}, \mathbf{q} ; i \Omega_{N}\right)$. Let us note that they possess the following symmetry,

$$
\begin{aligned}
& I_{r s}^{e f}\left(\mathbf{k}, \mathbf{q} ; i \Omega_{N}\right)=I_{s r}^{f e}\left(\mathbf{q}, \mathbf{k} ; i \Omega_{N}\right) \\
& J_{r s}^{e f}\left(\mathbf{k}, \mathbf{q} ; i \Omega_{N}\right)=J_{s r}^{f e}\left(\mathbf{q}, \mathbf{k} ; i \Omega_{N}\right) .
\end{aligned}
$$

[1] D. M. Eagles, Phys. Rev. 186, 456 (1969).

[2] A. J. Leggett, J. Phys. Colloques 41, 19 (1980).

[3] C. A. Regal, M. Greiner, and D. S. Jin, Phys. Rev. Lett. 92, 040403 (2004), cond-mat/0401554.

[4] M. Bartenstein, A. Altmeyer, S. Riedl, S. Jochim, C. Chin, J. Hecker Denschlag, and R. Grimm, Phys. Rev. Lett. 92, 120401 (2004), cond-mat/0401109.

[5] M. W. Zwierlein, C. A. Stan, C. H. Schunck, S. M. F. Raupach, A. J. Kerman, and W. Ketterle, Phys. Rev. Lett. 92, 120403 (2004), cond-mat/0403049.

[6] T. Bourdel, L. Khaykovich, J. Cubizolles, J. Zhang, F. Chevy, M. Teichmann, L. Tarruell, S. J. J. M. F. Kokkelmans, and C. Salomon, Phys. Rev. Lett. 93, 050401 (2004), cond-mat/0403091.

[7] P. Nozières and S. Schmitt-Rink, J. Low Temp. Phys. 59, 195 (1985).

[8] M. W. J. Romans and H. T. C. Stoof (2006), condmat/0607045.

[9] R. Haussmann, Phys. Rev. B49, 12975 (1994).

[10] R. Haussmann, W. Rantner, S. Cerrito, and W. Zwerger, Phys. Rev. A75, 023610 (2007), cond-mat/0608282.

[11] V. M. Loktev, R. M. Quick, and S. G. Sharapov, Phys. Rept. 349, 1 (2001).

[12] Q. Chen, J. Stajic, S. Tan, and K. Levin, Phys. Rept. 412, 1 (2005).

[13] R. B. Diener, R. Sensarma, and M. Randeria (2007), arXiv:0709.2653 [cond-mat.other].
[14] J. Carlson, S.-Y. Chang, V. R. Pandharipande, and K. E. Schmidt, Phys. Rev. Lett. 91, 050401 (2003), physics/0303094.

[15] E. Burovski, N. Prokof'ev, B. Svistunov, and M. Troyer, Phys. Rev. Lett. 96, 160402 (2006), cond-mat/0602224.

[16] Y. Nambu and G. Jona-Lasinio, Phys. Rev. 122, 345 (1961)

[17] Y. Nambu and G. Jona-Lasinio, Phys. Rev. 124, 246 (1961).

[18] B. C. Barrois, Nucl. Phys. B129, 390 (1977).

[19] S. C. Frautschi (1978), presented at Workshop on Hadronic Matter at Extreme Energy Density, Erice, Italy, Oct 13-21, 1978.

[20] M. G. Alford, A. Schmitt, K. Rajagopal, and T. Schaefer (2007), arXiv:0709.4635 [hep-ph].

[21] E. Babaev, Int. J. Mod. Phys. A16, 1175 (2001), hepth/9909052.

[22] P. Castorina, G. Nardulli, and D. Zappala, Phys. Rev. D72, 076006 (2005), hep-ph/0505089.

[23] E. Babaev, Phys. Rev. D62, 074020 (2000), hep$\mathrm{ph} / 0006087$.

[24] S. J. Hands, J. B. Kogut, and C. G. Strouthos, Phys. Lett. B515, 407 (2001), hep-lat/0107004.

[25] M. Kitazawa, T. Koide, T. Kunihiro, and Y. Nemoto, Phys. Rev. D70, 056003 (2004), hep-ph/0309026.

[26] M. Matsuzaki, Phys. Rev. D62, 017501 (2000), hepph/9910541. 
[27] H. Abuki, T. Hatsuda, and K. Itakura, Phys. Rev. D65, 074014 (2002), hep-ph/0109013.

[28] K. Nawa, E. Nakano, and H. Yabu, Phys. Rev. D74, 034017 (2006), hep-ph/0509029.

[29] M. Kitazawa, D. H. Rischke, and I. A. Shovkovy (2007), arXiv:0709.2235 [hep-ph].

[30] D. Ebert, K. G. Klimenko, and V. L. Yudichev, Phys. Rev. C72, 015201 (2005), hep-ph/0412129.

[31] D. Ebert, K. G. Klimenko, and V. L. Yudichev, Eur. Phys. J. C53, 65 (2008), arXiv:0705.2666 [hep-ph].

[32] J. Deng, A. Schmitt, and Q. Wang, Phys. Rev. D76, 034013 (2007), nucl-th/0611097.

[33] L. He and P. Zhuang, Phys. Rev. D76, 056003 (2007), arXiv:0705.1634 [hep-ph].

[34] G.-F. Sun, L. He, and P. Zhuang, Phys. Rev. D75, 096004 (2007), hep-ph/0703159.

[35] Y. Nishida and H. Abuki, Phys. Rev. D72, 096004 (2005), hep-ph/0504083.

[36] H. Abuki, Nucl. Phys. A791, 117 (2007), hep$\mathrm{ph} / 0605081$.

[37] L. He and P. Zhuang, Phys. Rev. D75, 096003 (2007), hep-ph/0703042.

[38] M. Buballa, Phys. Rept. 407, 205 (2005), hep$\mathrm{ph} / 0402234$.

[39] D. H. Rischke, Prog. Part. Nucl. Phys. 52, 197 (2004).

[40] H. B. Nielsen and S. Chadha, Nucl. Phys. B105, 445 (1976).

[41] D. Blaschke, D. Ebert, K. G. Klimenko, M. K. Volkov, and V. L. Yudichev, Phys. Rev. D70, 014006 (2004), hep-ph/0403151.

[42] T. Brauner, Phys. Rev. D75, 105014 (2007), hep$\mathrm{ph} / 0701110$.
[43] E. Abrahams and T. Tsuneto, Phys. Rev. 152, 416 (1966).

[44] T. Schaefer, D. T. Son, M. A. Stephanov, D. Toublan, and J. J. M. Verbaarschot, Phys. Lett. B522, 67 (2001), hep-ph/0108210.

[45] D. J. Thouless, Ann. Phys. 10, 553 (1960).

[46] J. O. Andersen, Rev. Mod. Phys. 76, 599 (2004), condmat/0305138.

[47] T. Brauner, Phys. Rev. D72, 076002 (2005), hep$\mathrm{ph} / 0508011$.

[48] A. Schmitt, I. A. Shovkovy, and Q. Wang, Phys. Rev. D73, 034012 (2006).

[49] For sake of an easy comparison, we closely follow the notation of Abuki 36]. The only important difference is that we define the Nambu doublet with the charge conjugate spinor $\psi^{\mathcal{C}}$ instead of the simple Dirac conjugate $\bar{\psi}^{T}$.

[50] Suppose that we integrate in $D$ dimensions a function $f(\mathbf{k})$ with asymptotics $f(\mathbf{k}) \sim 1 /|\mathbf{k}|^{n}$ as $|\mathbf{k}| \rightarrow \infty$, with integer $n$. A sharp cutoff restricts the integration domain to a sphere of radius $\Lambda$. Shifting the integration variable by a small vector $\mathbf{a}$ is equivalent to changing the integration domain by a thin shell (of size $\sim|\mathbf{a}|$ ). The corresponding change of the integral is estimated by the value of the function on the shell times its volume, i.e., $\left(1 / \Lambda^{n}\right) \Lambda^{D-1}|\mathbf{a}|=|\mathbf{a}| \Lambda^{D-n-1}$. This will be negligible (and the shift thus permitted) provided $n \geq D$.

[51] The scalar field $\Delta_{a}$ is a singlet of the original flavor SU(2) symmetry so that this is no more a symmetry of the bosonized action (3). The flavor quantum number has been "integrated out" together with the quarks. 